\title{
Negative ion production near a divertor plate
}

\author{
F. Taccogna ${ }^{a^{*}}$, R. Schneider ${ }^{\mathrm{a}}$, K. Matyash ${ }^{\mathrm{a}}$ S. Longo ${ }^{\mathrm{b}}$, M. Capitelli ${ }^{\mathrm{b}}$ and D. Tskhakaya ${ }^{\mathrm{c}}$ \\ ${ }^{a}$ Max Planck Institute für Plasmaphysik, EURATOM Association, Wendelsteinstrasse 1, D- 17491 \\ Greifswald, Germany. \\ ${ }^{b}$ Dip. di Chimica, Universita' di Bari and IMIP-CNR Sect. Bari, via Orabona 4, Bari,I-70126, Italy \\ ${ }^{c}$ Department of Plasma Physics, Andronikashvili Institue of Physics, Tamarashvili str.6, 0177 Tbilisi, \\ Georgia
}

\begin{abstract}
In detached divertor operation, typical plasma parameters in front of target plates are densities from $10^{19} \mathrm{~m}^{-3}$ up to $10^{20} \mathrm{~m}^{-3}$ and temperatures below $3 \mathrm{eV}$. In this regime, production of negative hydrogen ions can occur, mainly through vibrational excited molecule or by negative surface ionization of hydrogen ions. To study this effect, we have developed a 1D3V Particle-in-Cell electrostatic model of the electro-negative sheath. The motion of charged particles $\left(\mathrm{e}, \mathrm{H}^{+}, \mathrm{H}_{2}{ }^{+}\right.$and $\left.\mathrm{H}^{-}\right)$in their self-consistent electric field and of excited neutral-particle states $\left(\mathrm{H}(\mathrm{n}=1,2,3)\right.$ and $\left.\mathrm{H}_{2}\left(\mathrm{X}^{1} \Sigma_{\mathrm{g}}^{+}, v=0, \ldots, 14\right)\right)$ is simulated. Surface and volumetric processes have been included by using different Monte Carlo Collision methods. The importance of negative ions for the sheath potential and hydrogen fluxes on to the divertor will be calculated for different parameters.
\end{abstract}

PACS: $\quad$ 52.25.Dg, 52.25.Ya, 52.40.Hf

JNM keywords: $\quad$ D0500, H0400, P0500, S1300, T0100

PSI-16 keywords: $\quad$ Divertor modelling, Hydrogen, Neutral modelling, Recycling, Sheaths

*Corresponding author address: $\quad$ Wendelsteinstrasse 1, Greifswald, D-1749, Germany

"Corresponding author E-mail: $\quad$ taccogna@ipp.mpg.de

Presenting author: $\quad$ Francesco Taccogna

Presenting author E-mail: $\quad$ taccogna@ipp.mpg.de 


\section{Introduction}

The divertor is considered to be the most reliable concept for a tokamak reactor from the viewpoints of neutral particle control and heat flux density reduction [1].

One of the characteristic features of the transition between plasma and an absorbing wall is the build-up of an electric space-charge potential at the plasma edge. In tokamaks, this boundary layer is interspersed with a magnetic field hitting wall at some angle. Thereby, the sheath has been shown to be composed of "magnetic" and "Debye" regions. The relatively large width of the magnetic sheath (hundreds of Debye lenghts) permits particle interactions with surface-emitted material to be potentially significant and affecting particle, heat transport and the sheath parameters themselves. Among the different processes involved in the divertor region, the negative ion $\mathrm{H}^{-}$production could be important due to the fact that negative ions influence the sheath structure and subsequently the transport properties and the boundary layer dynamics including impurities.

\subsection{Production and Destruction of $\mathbf{H}^{-}$}

In detached divertor operation, typical plasma parameters in front of a divertor plate are electron densities from $10^{19} \mathrm{~m}^{-3}$ up to $10^{20} \mathrm{~m}^{-3}$ and temperatures below $3 \mathrm{eV}$ [1]. In this regime, production of negative hydrogen ions can occur in the volume by dissociative attachment to vibrational excited hydrogen molecules (reaction (8) in Tab. I). The vibrational levels $v>4$ provide the main contribution to the negative ion formation via vibrationally excited molecules. Additionally, negative ions are produced on the surface in two different ways: by energetic hydrogen atoms and molecules bouncing back as $\mathrm{H}^{-}$or by ionic processes. The latter is a favorable process due to the fact that positive ions are accelerated in the sheath near the wall by the potential drop. For the destruction of negative ions the main mechanisms are electron detachment in collisions with electrons (reaction (12)), recombination with the 
two types of positive ions (reactions $(17,21)$ ), and electron detachment in collisions with atoms and molecules (reactions (22-23)).

Since processes at the plasma-wall boundary are rather complex, it is desirable to simulate these processes in a numerical model in order to obtain insight into the relative importance and interaction of different effects. Fluid models [1,2] have usually been applied for analysis of divertor plasmas, but the usual fluid equations are not suitable to describe this region due to its kinetic and non-Maxwellian character. At the same time, kinetic simulations [3] of the divertor region usually include just the plasma (not negative ions), so that neutral transport is not considered at all.

In this paper, we aim to study the importance of collisions in a divertor plasma. For this, we developed a 1D-3V electrostatic Particle-in-Cell (PIC) code. Different Monte Carlo Collision (MCC) techniques have been used to model plasma-plasma and plasma-neutral interactions. Plasma recycling, secondary electrons and neutral particles are self-consistently included in the simulation model. In the next section, we explain the models used in the code. Results of simulations are shown in section 3.

\section{Numerical Model}

The approach we follow is to solve self-consistently the kinetic equations for the electrons and ions $\left(\mathrm{H}^{+}, \mathrm{H}_{2}^{+}, \mathrm{H}^{-}\right)$by the PIC method [4]. Neutral particle emission at the surface and transport of neutrals within the sheath are modelled using data/models for hydrogen ions and neutrals reflection, dissociation and recombination processes [12-14]. Charged and neutral particles dynamics are numerically solved in different modules coupled explicitly.

\subsection{PIC Module}

The complete motion of electrons and ions in the self-consistent electric and applied magnetic fields (forming an angle $\theta=85^{\circ}$ with the normal to the surface) is calculated by 
solving the equation of motion, computing collisional effects via Monte Carlo, and using a space grid for the potential calculation, as in a typical PIC cycle.

In order to avoid the injection problem, the plasma is bounded between two conducting walls, reflecting schematically the poloidal profile of the tokamak. An ambipolar particle source (electrons and protons) is located in the centre of the system and is of finite size (see Fig. 1), so that quasi-neutrality near the source region is self-consistently maintained by the plasma itself [4].

In the code both charged-neutral particle and Coulomb collisions (see Tab. I for the list of collisions) are implemented. For charged-neutral particle collisions the null collision method is used [8], which ensures high accuracy and high speed of the calculations, for coulomb collisions the cumulative small-angle Monte Carlo methodology [9] is used and for chargedcharged particle collisions a Direct Simulation Monte Carlo technique [10] is used. Within the PIC module, only charged particles are moved and the code does not follow the dynamics of the neutrals but rather treats the latter as a fixed background with density and velocities profiles from the previous iteration of the neutral module.

When a particle hits the wall different processes are possible (see Tab. II for the list of processes occurring). Secondary electron emission from the wall is included following [11]. While, when ions reach the surface, among the different processes [12] occurring (reflection, neutralization, recombination, dissociation, etc.), the activation of high vibrational states [13] and the formation of negative ions by an electron capture from the wall [14] are included as directly related to the negative ion production. A small pump exhaust coefficient $\left(\gamma_{\text {pump }}=0.05\right)$ is used.

\subsection{Neutral Module}

Every 1000 PIC cycles (in order to allow the ion system to relax), the neutral module is called. Neutral pseudo-particles are launched from the surface (according to the averaged ion 
particle flux calculated during the PIC module) with atomic electronic and molecular vibrational distributions depending on the averaged ion energy flux to the wall. The first three electronic states of $\mathrm{H}(\mathrm{n}=1,2,3)$ and fifteen vibrational states of the fundamental level of $\mathrm{H}_{2}$ $\left(\mathrm{X}^{1} \Sigma_{\mathrm{g}}^{+}, v=0, \ldots, 14\right)$ are taken into account. Their initial velocity distribution is taken to be halfMaxwellian with a temperature of typically $1000 \mathrm{~K}$. All the collisions considered in Tab. I and involving neutrals are processed using values of plasma densities and velocities averaged on the previous PIC module. The balance takes into account that the lowest $(v=0-6)$

vibrational levels are essentially populated by eV collisions (reaction (3) in Tab. I). The higher levels are essentially populated by EV processes (reaction (4) in Tab. I) that means through electron impact excitation to the $B^{1} \Sigma_{u}^{+}$and $C^{1} \Pi_{u}$ electronic states, followed by radiative decay to vibrationally excited molecules at the electronic ground state. Neutralneutral collisions may be ignored, assuming a neutral free molecular regime (the mean free path is much longer than the dimension of the simulated system). The losses of the vibrationally excited molecules via deactivation at the walls are also included in the model [15].

\section{Results}

Solutions were obtained for various plasma source parameters. All results presented in this paper are for a magnetic field $\mathrm{B}=1 \mathrm{~T}$ (the change of the absolute value of $\mathrm{B}$ will only change the length scale not the distribution of species). The simulation system has lengths of about $\mathrm{L}=50 \varrho_{\mathrm{i}}$, where $\varrho_{\mathrm{i}}$ is the ion gyroradius, in order to resolve the magnetic pre-sheath. All the profiles showed are averaged over a few tens of plasma oscillation periods. On the left it is represented the source region, while the wall is located on the right.

Three different cases of plasma parameters in the source region are considered:

1) $\mathrm{n}_{0}=1 \times 10^{18} \mathrm{~m}^{-3}, \mathrm{~T}_{0}=40 \mathrm{eV}$; 
2) $\mathrm{n}_{0}=1 \times 10^{19} \mathrm{~m}^{-3}, \mathrm{~T}_{0}=20 \mathrm{eV}$;

3) $\mathrm{n}_{0}=1 \times 10^{20} \mathrm{~m}^{-3}, \mathrm{~T}_{0}=2 \mathrm{eV}$;

Here, cases (2) and (3) can be considered as studies of attached and detached plasma in front of a divertor plate. Case (1) represents areas away from the separatrix with low heat fluxes and densities, but still relatively high plasma temperatures. These conditions are typical for baffle regions in V-shaped geometries like in Asdex-Upgrade [1].

Regarding the production of negative ions, the most interesting case is the detached divertor case (3), where the dominant dependence is the electron temperature. Indeed, as shown in Fig. 2.a, the percentage of negative ions density reaches a value of $10 \%$ of the electron density in the divertor region, while in the other two cases it is less then 5\%. For this reason we present plots concerning the case (3). In the cases (1) and (2), the electron temperature is greater then $10 \mathrm{eV}$, and the destruction mechanism of negative ions by electron detachment (reaction (12) in Tab. I) is very effective due to the fact that the cross section has a peak at $\varepsilon \approx 15 \mathrm{eV}$.

In the code, we are able to distinguish between the different electrons (volume or surface produced). The secondary electrons emitted from the wall represent only $10 \%$ of the total electron population, while the electron density on the wall is reduced of two order of magnitude respect to the source region. Consequently, the sheath has a monotonic classical behavior and no double layer structure appears near the wall. The $\mathrm{H}^{+}$density drops monotonically allowing the separation of the magnetic pre-sheath and the electrostatic sheath. On the wall, the electrostatic sheath results in the electron density being 5 times less than the positive ion density. The molecular ion density represents just $8 \%$ of the positive ion density. Indeed, due to the low value of electron temperature (Fig. 2.b), the ionization of molecules (ionization potential $\mathrm{I}=16 \mathrm{eV}$ ) becomes a rare process. The negative ion density behavior reflects the presence of two different components, volume-produced and surface-produced. 
The first is dominant in the source region, where the electron temperature is high enough to excite vibrational levels (see Fig. 2.d) necessary to produce negative ions by electron attachment. At the same time, the electron temperature is low enough to reduce the destruction processes by electron detachment. The heating of electrons in the source region $\left(\mathrm{T}_{\mathrm{e}}>\mathrm{T}_{0}\right)$ is due to electronic and vibrational de-excitation of molecules.

The temperature of the negative ions (Fig. 2.b) shows an increase in the sheath due to the acceleration of surface produced $\mathrm{H}^{-}$from the wall to the bulk. The temperature of the negative ions in the pre-sheath drops to the temperature of the positive ions due to thermalization.

The atomic densities are shown in Fig. 2.c. The electronic state emitted from the wall is the fundamental $n=1$. In the sheath, the energy of the electrons is sufficient to excite the atom up to the first excited state $n=2$, while the second is almost irrelevant $(\Delta \varepsilon=12.09 \mathrm{eV})$. The densities of the excited states reach their maximum in the magnetic pre-sheath region where the neutral density and electron energy are high enough. Near the wall, the neutral density for $\mathrm{n}=1$ is high, but the electron energy is not sufficient to produce excited electronic states. The double peak of $n=3$ is a simple statistical effect due to small number of particles with $n=3$.

The molecular density behavior is shown in Fig. 2.d. It is evident that the densities of the vibrational excited states $(v>2)$ are the sum of two curves, one representing the emission from the wall (peaked to the vibrational level $v=0$ due to the low temperature of this case) and the other representing the bulk excitation by electrons populating the levels $5<v<8$ responsible for the high negative ion density revealed for this case.

\section{Conclusion}

We have studied the divertor plasma in front of a target plate by a one-dimensional electrostatic particle code. Special interest was on the production of negative ions by electron attachment through vibrational excited molecule or by negative surface ionization of 
hydrogen ions. The maximum production of negative ions is obtained from the detached divertor case with lowest electron temperature (about $2 \mathrm{eV}$ ), where the electrons have sufficient energy to form vibrational excited levels, but not enough to destruct effectively by electron detachment. It is shown that the negative ion $\mathrm{H}^{-}$production influences the sheath structure and subsequently the transport properties and the boundary layer dynamics.

\section{Acknowledgements}

This work is supported by the European Community under the MARIE CURIE INTRAEUROPEAN FELLOWSHIP: "Negative ion source model" and by MIUR-PRIN 2005 under the contract n. 2005039049_005: "Dinamica dei processi elementary per la chimica e la fisica dei plasmi”. R. Schneider acknowledges funding of the work by the Initiative and Networking Fund of the Helmholtz Association. 


\section{References}

[1] R. Schneider, X. Bonnin, K. Borrass, D. P. Coster, H. Kastelewicz, D. Reiter, V. A. Rozhansky, B. J. Braams, Contr. Plasma Phys. 46(1-2) (2006) 3.

[2] W. M. Stacey, Phys. Plasmas 5(4) (1998) 1015.

[3] T. Q. Hua, J. N. Brooks, Phys. Plasmas 1(11) (1994) 3607.

[4] D. Tskhakaya, S. Kuhn, Plasma Phys. Control. Fusion 47 (2005) A327.

[5] R. Celiberto, R. K. Janev, A. Laricchiuta, M. Capitelli, J. M. Wadehra, D. E. Atems, Atomic Data and Nuclear Data Tables 77 (2001) 161.

[6] R. K. Janev, W. D. Longer, K. Evants, Jr., D. E. Post, Elementary Processes in Hydrogen-Helium Plasmas (Springer, Berlin, 1987).

[7] C. F. Barnett, Atomic Data for Fusion, ORNL- 6068 Vol. I (Ed. By Atomic Data Center, ORNL, Tennessee, 1990).

[8] V. Vahedi, M. Surendra, Comp. Phys. Comm. 87 (1995) 179.

[9] K. Nanbu, Phys. Rev. E 55(4) (1997) 4642.

[10] K. Nanbu, IEEE Trans. Plasma Sci. 28(3) (2000) 971.

[11] M. A. Furman, M. T. F. Pivi, Phys. Rev. Spec. Top. - Accel. \& Beams 5 (2002) 124404.

[12] W. Eckstein, H. Werbeek, Nucl. Fus., Spec. Issue IAEA (1984) 12.

[13] M. Seidl, H. L. Cui, J. D. Isenberg, H. J. Kwon, B. S. Lee, S. T. Melnychuk, J. Appl. Phys. 79(6) (1996) 2896.

[14] J. R. Hiskes, A. M. Karo, J. Appl. Phys. 67(11) (1990) 6621.

[15] M. Cacciatore, G. D. Billing, Pure and Appl. Chem. 68(5) (1996) 1075. 


\begin{tabular}{|c|c|c|}
\hline N. & Volume Process & Reference \\
\hline 1 & $e+H(n) \quad e+H(m)$ & {$[5]$} \\
\hline 2 & $\mathrm{e}+\mathrm{H}(\mathrm{n}) \quad 2 \mathrm{e}+\mathrm{H}^{+}$ & {$[5]$} \\
\hline 3 & $\mathrm{e}+\mathrm{H}_{2}(v) \quad \mathrm{e}+\mathrm{H}_{2}(w)$ & {$[5]$} \\
\hline 4 & $\mathrm{e}+\mathrm{H}_{2}(v) \quad \mathrm{e}+\mathrm{H}_{2}(w)+\mathrm{h} v$ & {$[5]$} \\
\hline 5 & $\mathrm{e}+\mathrm{H}_{2}(v) \quad 2 \mathrm{e}+\mathrm{H}_{2}^{+}$ & {$[5]$} \\
\hline 6 & $\mathrm{e}+\mathrm{H}_{2}(v) \quad \mathrm{e}+\mathrm{H}(\mathrm{n})+\mathrm{H}(\mathrm{m})$ & {$[5]$} \\
\hline 7 & $\mathrm{e}+\mathrm{H}_{2}(v) \quad 2 \mathrm{e}+\mathrm{H}(\mathrm{n})+\mathrm{H}^{+}$ & {$[5]$} \\
\hline 8 & $\mathrm{e}+\mathrm{H}_{2}(v) \quad \mathrm{H}(\mathrm{n})+\mathrm{H}^{-}$ & {$[5]$} \\
\hline 9 & $\mathrm{e}+\mathrm{H}_{2}^{+} \quad \mathrm{e}+\mathrm{H}^{+}+\mathrm{H}(\mathrm{n})$ & {$[6]$} \\
\hline 10 & $\mathrm{e}+\mathrm{H}_{2}^{+} \quad \mathrm{H}(\mathrm{n})+\mathrm{H}(\mathrm{m})$ & {$[6]$} \\
\hline 11 & $\mathrm{e}+\mathrm{H}_{2}^{+} \quad 2 \mathrm{e}+2 \mathrm{H}^{+}$ & {$[6]$} \\
\hline 12 & $\mathrm{e}+\mathrm{H}^{-} \quad 2 \mathrm{e}+\mathrm{H}(\mathrm{n})$ & {$[6]$} \\
\hline 13 & $\mathrm{H}^{+}+\mathrm{H}(\mathrm{n}) \quad \mathrm{H}(\mathrm{n})+\mathrm{H}^{+}$ & [6] \\
\hline 14 & $\mathrm{H}^{+}+\mathrm{H}_{2}(v) \quad \mathrm{H}^{+}+\mathrm{H}_{2}(w)$ & {$[6]$} \\
\hline 15 & $\mathrm{H}^{+}+\mathrm{H}_{2}(v) \quad \mathrm{H}_{2}^{+}+\mathrm{H}(\mathrm{n})$ & {$[6]$} \\
\hline 16 & $\mathrm{H}^{+}+\mathrm{H}_{2}(v) \quad \mathrm{H}^{+}+\mathrm{H}(\mathrm{n})+\mathrm{H}(\mathrm{m})$ & {$[6]$} \\
\hline 17 & $\mathrm{H}^{+}+\mathrm{H}^{-} \quad \mathrm{H}(\mathrm{n})+\mathrm{H}(\mathrm{m})$ & {$[6]$} \\
\hline 18 & $\mathrm{H}_{2}^{+}+\mathrm{H}(\mathrm{n}) \quad \mathrm{H}^{+}+\mathrm{H}_{2}(v)$ & {$[7]$} \\
\hline 19 & $\mathrm{H}_{2}^{+}+\mathrm{H}_{2}(v) \quad \mathrm{H}_{2}(w)+\mathrm{H}_{2}^{+}$ & {$[6]$} \\
\hline 20 & $\mathrm{H}_{2}^{+}+\mathrm{H}_{2}(v) \quad \mathrm{H}^{+}+\mathrm{H}(\mathrm{n})+\mathrm{H}_{2}(v)$ & {$[6]$} \\
\hline 21 & $\mathrm{H}_{2}^{+}+\mathrm{H}^{-} \quad \mathrm{H}_{2}(v)+\mathrm{H}(\mathrm{n})$ & {$[6]$} \\
\hline 22 & $\mathrm{H}^{-}+\mathrm{H}(\mathrm{n}) \quad \mathrm{H}_{2}(v)+\mathrm{e}$ & {$[6]$} \\
\hline 23 & $\mathrm{H}^{-}+\mathrm{H}_{2}(v) \quad \mathrm{H}(\mathrm{n})+\mathrm{H}_{2}(v)+\mathrm{e}$ & {$[6]$} \\
\hline
\end{tabular}

Table I - List of the most significant volume processes included in the model. 


\begin{tabular}{|c|c|c|c|c|}
\hline Colliding particle & \multicolumn{3}{|l|}{ Surface Process } & Reference \\
\hline \multirow[t]{3}{*}{ Electron } & \multicolumn{3}{|c|}{ - backscattered electrons } & {$[11]$} \\
\hline & \multicolumn{3}{|c|}{ - re-diffused electrons } & [11] \\
\hline & \multicolumn{3}{|c|}{ - true secondary electrons } & [11] \\
\hline \multirow[t]{5}{*}{$\mathrm{H}^{+}$} & \multicolumn{3}{|l|}{ - absorption } & [12] \\
\hline & \multirow[t]{4}{*}{ - reflection } & \multicolumn{2}{|c|}{$-\mathrm{H}^{+}$} & {$[12]$} \\
\hline & & \multirow[t]{2}{*}{ - neutralization } & - 1e-capture: H(n) & {$[13]$} \\
\hline & & & - 2e-capture: $\mathrm{H}^{-}$ & {$[13]$} \\
\hline & & & - recombination: $\mathrm{H}_{2}(w)$ & {$[14]$} \\
\hline \multirow[t]{8}{*}{$\mathrm{H}_{2}^{+}$} & \multicolumn{3}{|l|}{ - absorption } & {$[12]$} \\
\hline & \multirow{3}{*}{ - partial absorption } & \multicolumn{2}{|l|}{$-\mathrm{H}^{+}$} & {$[12]$} \\
\hline & & \multicolumn{2}{|c|}{ - 1e-capture: H(n) } & {$[13]$} \\
\hline & & \multicolumn{2}{|c|}{ - 2e-capture: $\mathrm{H}^{-}$} & {$[13]$} \\
\hline & \multirow{4}{*}{$\begin{array}{l}-\mathrm{H}_{2}^{+} \\
- \text {diss } \\
- \text { neu }\end{array}$} & & & {$[12]$} \\
\hline & & ciation: $\mathrm{H}$ & $+\mathrm{H}(\mathrm{n})$ & {$[12]$} \\
\hline & & alization & $-\mathrm{H}_{2}(w)$ & {$[14]$} \\
\hline & & & - dissociation: $H(n)+H(m)$ & {$[14]$} \\
\hline \multirow[t]{4}{*}{$\mathrm{H}(\mathrm{n})$} & \multicolumn{3}{|l|}{ - absorption } & {$[15]$} \\
\hline & \multirow[t]{3}{*}{ - reflection } & \multicolumn{2}{|c|}{$-H(n)$} & {$[15]$} \\
\hline & & \multicolumn{2}{|c|}{ - 1e-capture: $\mathrm{H}^{-}$} & {$[15]$} \\
\hline & & \multicolumn{2}{|c|}{ - recombination: $\mathrm{H}_{2}(w)$} & {$[15]$} \\
\hline \multirow[t]{5}{*}{$\mathrm{H}_{2}(v)$} & \multicolumn{3}{|l|}{ - absorption } & {$[15]$} \\
\hline & \multirow[t]{2}{*}{ - partial absorption } & $-H(n)$ & & {$[15]$} \\
\hline & & - 1e-cap & are: $\mathrm{H}^{-}$ & {$[15]$} \\
\hline & \multirow[t]{2}{*}{ - reflection } & \multicolumn{2}{|c|}{$-\mathrm{H}_{2}(w)$} & [15] \\
\hline & & \multicolumn{2}{|c|}{ - dissociation: $H(m)+H(n)$} & {$[15]$} \\
\hline
\end{tabular}

Table II - List of the most significant surface processes included in the model. 


\section{List of Figure Captions}

Figure 1 Schematic of the simulation domain.

Figure 2.a Spatial profiles of plasma densities for the case 3): $\mathrm{n}_{0}=10^{20} \mathrm{~m}^{-3} ; \mathrm{T}_{0}=2 \mathrm{eV}$.

Figure 2.b Spatial profiles of temperature of electrons, positive ions $\mathrm{H}^{+}$and negative ions $\mathrm{H}^{-}$for the case 3): $\mathrm{n}_{0}=10^{20} \mathrm{~m}^{-3} ; \mathrm{T}_{0}=2 \mathrm{eV}$.

Figure 2.c Spatial profiles of the different electronic energy level of atomic density for the case 3): $\mathrm{n}_{0}=10^{20} \mathrm{~m}^{-3} ; \mathrm{T}_{0}=2 \mathrm{eV}$.

Figure 2.d Spatial profiles of the different vibrational energy level of molecular density for the case 3): $\mathrm{n}_{0}=10^{20} \mathrm{~m}^{-3} ; \mathrm{T}_{0}=2 \mathrm{eV}$. 


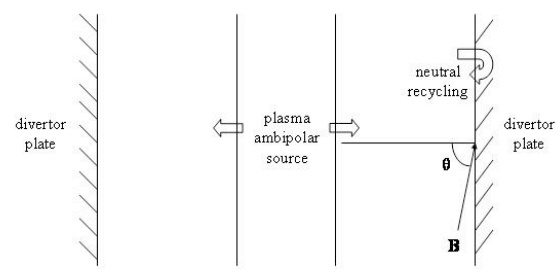

Fig. 1 - Taccogna et al. 


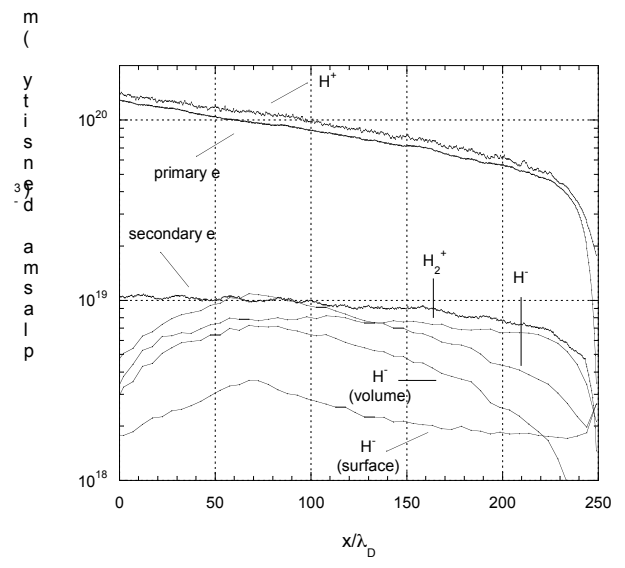

Fig. 2.a - Taccogna et al. 


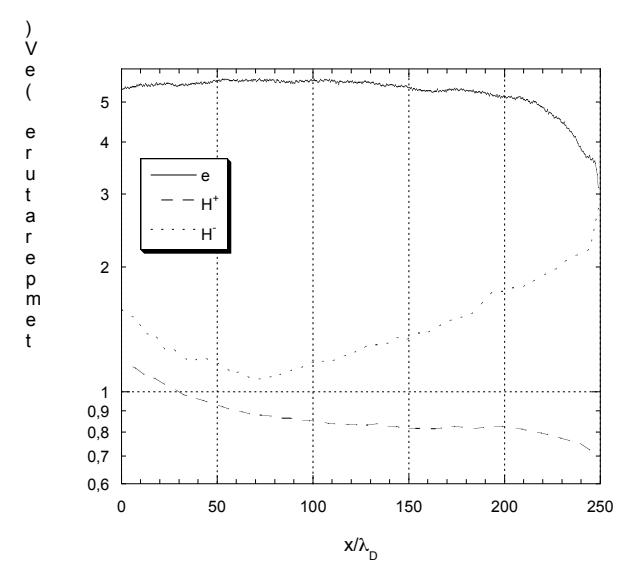

Fig. 2.b - Taccogna et al. 


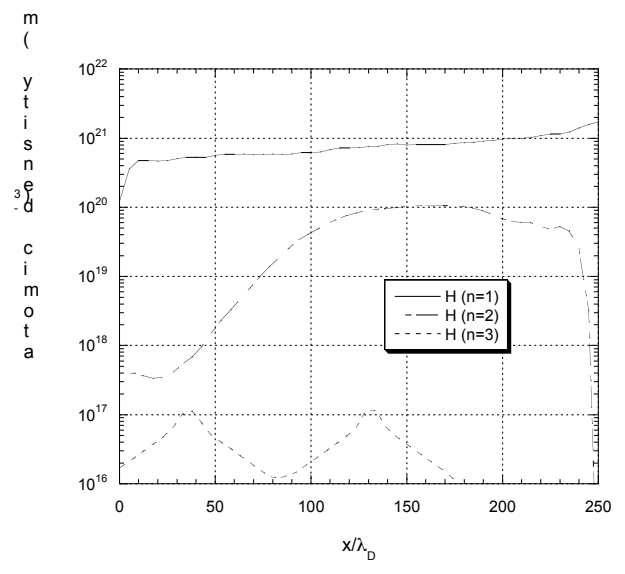

Fig. 2.c - Taccogna et al. 


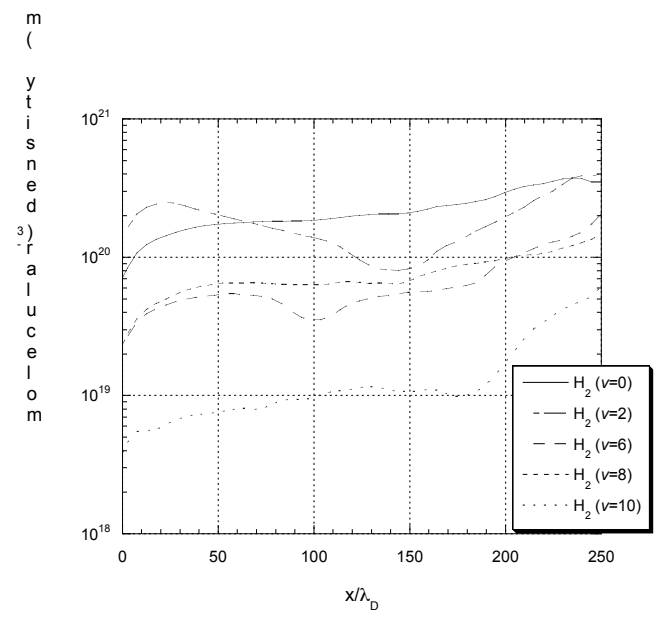

Fig. 2.d - Taccogna et al. 